\title{
Matrix effect on emission/current correlated analysis in laser-induced breakdown spectroscopy of liquid droplets
}

\author{
Jer-Shing Huang, Ching-Bin Ke, King-Chuen Lin* \\ Department of Chemistry, National Taiwan University, and Institute of Atomic and Molecular Sciences, Academia Sinica, Taipei 106, Taiwan, \\ $R O C$
}

Received 23 September 2003; accepted 15 December 2003

\begin{abstract}
We have investigated influence of matrix salts on the liquid droplets by laser-induced breakdown spectroscopy (LIBS). An electrospray ionization technique coupled with LIBS is employed to generate the microdroplets of the Na sample solution with various matrix salts added. A sequence of single-shot time-resolved LIB emission signals is detected. The LIB signal intensity integrated within a gate linearly correlates with the plasma-induced current response obtained simultaneously on a single-shot basis. The slopes thus obtained increase with the sample concentration, but appear to be irrespective of different matrix salts, added up to a $2000 \mathrm{mg} / \mathrm{l}$ concentration. The matrix salts involved have the same $\mathrm{K}^{+}$cation but different anions. Given a laser radiation emitting at $355 \mathrm{~nm}$ with the energy fixed at $23 \pm 1 \mathrm{~mJ}$, a limit of detection (LOD) of $1.0 \mathrm{mg} / 1 \mathrm{may}$ be achieved for the $\mathrm{Na}$ analysis. The current normalization might have probably taken into account the ablated amount of the sample and the plasma temperature. Accordingly, the LIB/current correlated analysis becomes efficient to suppress the signal fluctuation, improve the LOD determination, and concurrently correct the matrix effect.
\end{abstract}

(C) 2003 Elsevier B.V. All rights reserved.

Keywords: Laser-induced breakdown spectroscopy; Matrix effect; Electrospray ionization; Correlation method

\section{Introduction}

Laser-induced breakdown spectroscopy (LIBS) has grown to be a popular analytical technique for rapid analysis of a large variety of materials present in solids, gases, and liquids [1-11]. This technique provides the advantages of multi-elemental detection, non-invasive analysis, suitability to remote measurement, and minimal sample preparation. Nevertheless, it suffers from the emission signal fluctuation, which may restrict the LIBS sensitivity to some extent. However, the matrix composition contained in the analyzed materials has substantial impact on the accuracy of the elemental analysis. How to improve the signal quality and correct the matrix effect have been important issues in this field.

The signal fluctuation problem in the LIBS analysis are caused by complicated sources such as the laser pulse fluctuation, inconsistency of the sampled amount

\footnotetext{
*Corresponding author. Tel./fax: + 886-2-23621483.

E-mail address: kclin@ccms.ntu.edu.tw (K.-C. Lin).
}

of medium, instability of the plasma position, and the inverse bremsstrahlung radiation. Averaging the spectral signals over a number of laser shots is a way usually used to overcome this problem. Nevertheless, the improvement of the signal-to-noise ratio is offset by the non-linear optical ablation effect $[7,8]$. Internal standard method is an alternative solution via the measurement of the intensity ratio of analyte and reference element [6,9-11].

Another interference caused by the matrix effect has also been widely investigated to achieve matrix-corrected LIB emission signals [11-16]. For instance, Eppler et al. reported that in the sand and soil samples the LIB emission signals of $\mathrm{Pb}$ and $\mathrm{Ba}$ depend on the chemical speciation and the matrix composition [14]. They related the influence on the LIB measurement to plasma excitation conditions, the physical properties of the sample compound, matrix absorptivity, electron density, and plasma temperature. Such complicated factors may cause the accuracy of LIB measurement to be degraded if the calibration is not compound and matrix-specific. Chal- 
eard et al. quantized the LIB emission signals in air at atmospheric pressure by taking intensities of the emission lines as a function of the vaporized mass and the plasma excitation temperature [15]. Normalization of the LIB emission signals by these two parameters allowed for an efficient correction of matrix effects when the sample composition was changed. Hakkanen et al. studied the matrix effects in analyzing inhomogeneous paper coatings and found linear correlations between the LIB emission signals and the coat weight and the binder contents of the coatings [16]. Winefordner and coworkers investigated the influence of matrix effect on LIB signal of $\mathrm{Mg}$ in a powdered sample [17]. They suggested a method for matrix-free calibration based on normalization of the emission intensities by the surface densities of the analyzed materials. The surface density normalization method may account for the mechanical effect, in connection with the ablated weight, and is simpler than the correction with the electron temperature.

In the LIBS analysis of liquid samples, bulk liquid and liquid jet are frequently used as the sampling methods to minimize the signal fluctuation $[2,7,8]$. Different from these previous ones, in this work we use electrospray ionization needle to generate microdroplets of $\mathrm{NaCl}$ solution and then study the influence of a variety of matrix salts added on the resultant LIB emission signals [18-21]. Generation of microdroplets in the LIBS measurement may facilitate sample vaporization, atomization and excitation/ionization efficiency. In addition, as the microdroplets are ionized by LIBS, the current induced by the high voltage biased on the ionization needle may be feasibly collected [22]. To achieve matrix-corrected LIBS analysis, the LIBS and plasma-induced current signals are detected simultaneously on a single shot basis, upon laser irradiation onto the matrix-added microdroplets. We find that the intensities of single-shot time-resolved LIB emission may linearly correlate with the corresponding current intensities. The correlation plot yields a slope, which increases with the sample concentration, but appears almost independent of the matrix salts added up to a $2000 \mathrm{mg}$ / 1 concentration. Given the calibration curve thus obtained from the slopes, the limit of detection (LOD) for the $\mathrm{Na}$ analysis may reach $1 \mathrm{mg} / \mathrm{l}$, comparable to those reported previously [22]. The normalization of the LIB emission by the current may suppress the signal fluctuation, improve the subsequent LOD determination, and concurrently correct the matrix effect.

\section{Experimental setup}

\subsection{Laser radiation}

A schematic diagram for the LIBS system similar to this work has been illustrated elsewhere [22]. A third harmonic of Nd:YAG laser (DCR-2A, Spectra-Physics) emitting at $355 \mathrm{~nm}$ was used as the light source. The laser pulse was operated at $10 \mathrm{~Hz}$ with pulse duration 5-8 ns. The incident pulse was focused through a 10$\mathrm{cm}$ focal length quartz lens onto the microdroplets generated by an electrospray ionization needle. Throughout this work, the laser pulse energy prior to the quartz lens was controlled at $23 \pm 1 \mathrm{~mJ}$, which was monitored constantly with an energy meter (Model 210, Coherent). The laser-interacted spot was approximately $100-\mu \mathrm{m}$ diameter, but not measured precisely. The pulse-to-pulse fluctuation remained at $\pm 5 \%$.

\subsection{Electrospray ionization}

A homemade electrospray ionization device was employed to generate a stream of microdroplets, as described previously [22]. In brief, the analyte solution was syringe-pumped into a $200-\mu \mathrm{m}$ i.d. stainless steel spray needle. The needle served as the anode, biased at $3 \mathrm{kV}$, through which the analyte solution was sprayed toward the other metal base as the cathode. As carrier solvent evaporated, the analyte solution was then broken into droplets $[20,21]$. The laser pulse interacted with the sprayed droplets at $\sim 2 \mathrm{~mm}$ downstream from the needle tip. The irradiated spot was close to the tip of Taylor cone where a higher density of microdroplets may be gained $[20,21]$.

\subsection{LIB and current detection}

Upon laser-pulse interaction with the liquid droplets, the produced luminous plume was imaged with a set of $15-$ and $20-\mathrm{cm}$ focal length lenses onto a $0.35 \mathrm{~m}$ Czerney-Turner monochromator (Model 270, McPherson) behind which a photomultiplier tube (PMT, R955, Hamamatsu) was mounted. The LIBS emission was collected in perpendicular direction relative to the impinging laser beam. The grating in a monochromator, with a reciprocal linear dispersion of $2 \mathrm{~nm} / \mathrm{mm}$, was centered at $589 \mathrm{~nm}$ for the detection of the $\mathrm{Na}$ emission in the $3 \mathrm{p}^{2} \mathrm{P} \rightarrow 3 \mathrm{~s}^{2} \mathrm{~S}$ transition. Since the shape of Taylor cone in the electrospray ionization process was easily disturbed under ambient conditions, the irradiated spot size varied from shot to shot. To exempt from influence of such plume instability, the slits were open to $500-\mu \mathrm{m}$ wide, allowing transmission of the total plasma plume, which was magnified by a factor of 1.3 as imaged onto the entrance slit. Meanwhile, the slit width was narrow enough to substantially reduce the transmitted continuum background emission at the wavelength $589 \mathrm{~nm}$.

The obtained time-resolved LIB emission was fed into either a transient digitizer (Model 9450A, LeCroy) for single-shot profile recording or a boxcar integrator (Model SR250, Stanford Research System) for signal processing. The time-resolved spectra of the $\mathrm{Na}$ analyte 


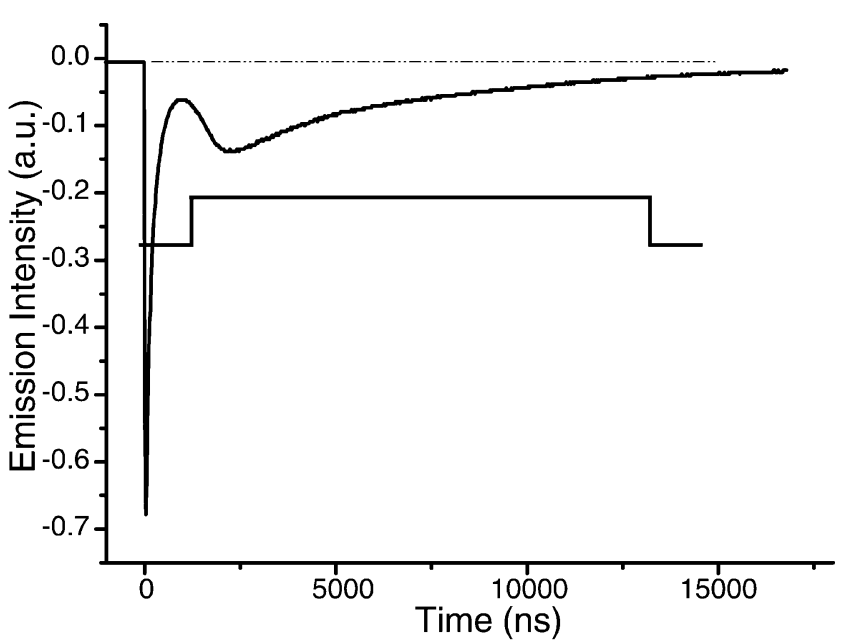

Fig. 1. Single-shot time dependent LIB spectra for a $200 \mathrm{mg} / 1 \mathrm{Na}$ solution. The spectrum within a gate, set at $12 \mu \mathrm{s}$ in width and delayed by $900 \mathrm{~ns}$ relative to the onset of continuum background emission, is integrated to obtain the intensity of LIB emission.

were integrated within a gate on a single-shot basis. In this work, the gate was opened in 900 ns delay relative to the onset of the continuum background emission. The LIB emission could then be the least interfered with by the intense continuum background. The gate width was optimized to $12 \mu \mathrm{s}$. The adjustment of the gate width and position may help suppress the LIB signal fluctuation to improve the signal-to-noise ratio.

The current was collected from the cathode via a high-pass filter into a second channel of either a transient digitizer or a boxcar integrator. It was monitored concurrently with the LIB emission. The current profile oscillated rapidly in a nanosecond scale, as displayed in the previous work [22]. Its first maximum peak was then integrated within a $20 \mathrm{~ns}$ gate throughout all the experiments. In fact, an appropriate selection of RC time constant may slow down the oscillation and extend the life time of each peak. To acquire a better LIB emission/current (LIB/C) correlation linearity, it must rely on an appropriate adjustment of the current gate width and position.

\subsection{Reagents}

The Na element as the analyte was made from its chloride solution. A $1000 \mathrm{mg} / \mathrm{l} \mathrm{NaCl}$ (Reagent grade, Merck) solution was first prepared with water/methanol $(\mathrm{v} / \mathrm{v}=1: 1)$ (HPLC grade, Baker) as solvent and then diluted to the desired concentrations. For gaining a better electrospray efficiency, we used a water/methanol $(\mathrm{v} / \mathrm{v}=1: 1)$ mixed solvent throughout this work. To study the matrix effect, the $\mathrm{KCl}, \mathrm{KNO}_{2}$, and $\mathrm{KH}_{2} \mathrm{PO}_{4}$ (Reagent grade, Merck), prepared at concentration from 100 to $2000 \mathrm{mg} / \mathrm{l}$, were added individually to the $\mathrm{NaCl}$ solution as the matrix salt.

\section{Results and discussion}

\subsection{Matrix effect on LIB emission/current correlation}

Three diatomic and polyatomic salts, $\mathrm{KCl}, \mathrm{KNO}_{2}$, and $\mathrm{KH}_{2} \mathrm{PO}_{4}$, were added individually as the matrix to the $\mathrm{NaCl}$ solution, and the generated microdroplets were irradiated by a laser pulse at $355 \mathrm{~nm}$. The resultant LIB emission signal of $\mathrm{Na}$ and the induced current were acquired concurrently up to 200 shots. Fig. 1 shows an example of single-shot time-dependent LIB emission spectrum of $\mathrm{Na}$ detected from the sample microdroplets. The spectrum is then integrated within a $12 \mu \mathrm{s}$ gate in 900 ns delay relative to the onset of the continuum background emission. The LIB emission intensity thus obtained can be linearly correlated with the plasmarelated current intensity. The correlation linearity relies on adjustment of the current gate width and position, as mentioned in Section 2. Fig. 2 shows comparison for the linearity acquired under different current gate conditions. The slope in the LIB/C correlation plot increases with the concentration of the analyte solution, as shown in Fig. 3. A possible interpretation for the sources leading to the LIB signal fluctuation is referred to our previous work [22]. Given the $\mathrm{NaCl}$ solution with $\mathrm{Na}$ concentration fixed at $200 \mathrm{mg} / \mathrm{l}$, Fig. 4 shows the slopes of LIB/C correlation plots as a function of 100, 500, and $1000 \mathrm{mg} / 1 \mathrm{KCl}, \mathrm{KNO}_{2}$, and $\mathrm{KH}_{2} \mathrm{PO}_{4}$ solutions added individually as matrix. The LIB/C slopes in the figure remain almost constant without influence of the matrix added. The figure also shows the results of blank solutions, which contain the same matrix solutions but without the sample added. Under otherwise identical

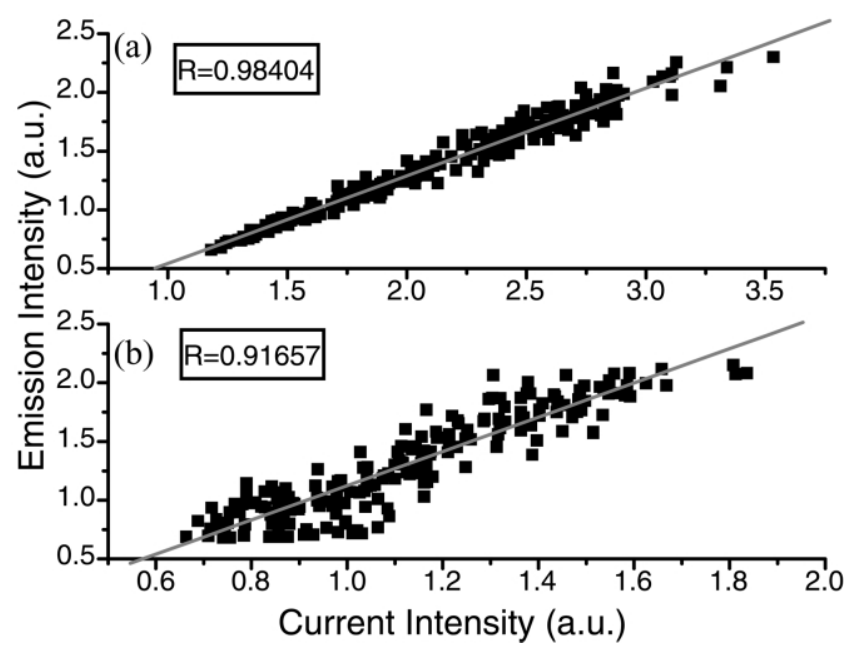

Fig. 2. Comparison of the correlation plots of Na LIB emission intensity vs. corresponding current intensity acquired with different gate conditions. (a) The first maximum peak of the current profile is gated optimally within a 20 ns duration; (b) The gate width remains the same, but the position is slightly shifted by $<5$ ns away from the onset of the current profile. 


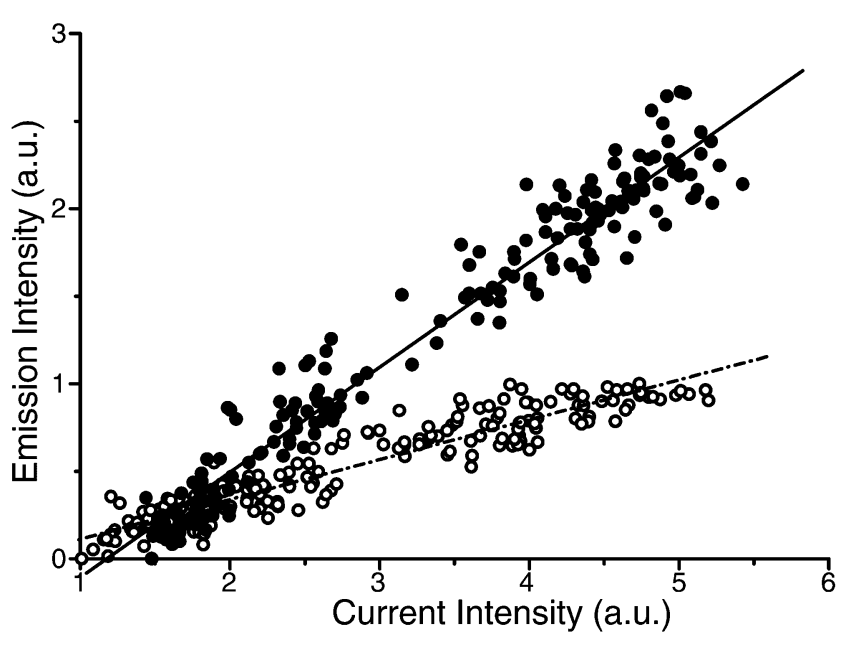

Fig. 3. The correlation plot of Na LIB emission intensity vs. corresponding current intensity obtained at the Na concentration of $10(\mathrm{O})$ and $200(\mathbf{)} \mathrm{mg} / \mathrm{l}$, respectively, with identical amount of matrix salt added.

conditions, the blank solutions give rise to a non-zero slope, probably caused by the contribution of the residual continuum background emission.

The calibration curve is represented by the plot of the obtained slopes vs. the corresponding sample concentrations. The subsequent calibration curves, given in Fig. 5 , exhibit insignificant difference between the cases without and with the matrix added up to $2000 \mathrm{mg} / \mathrm{l}$. In the LIB/C correlation plots, the obtained regression coefficients for the slopes are larger than 0.95 . The error bar represented in the calibration curve denotes the standard deviation of the slope in the correlation plot. Note that the LIB/C slopes are in arbitrary units, but Figs. 4 and 5 are not in the same scale, since both experiments were carried out in different days. The gate conditions have to be re-adjusted to achieve better correlation linearity in the LIB/C plots. Given the slope of the calibration curve and the standard deviation obtained from the slope in correlation plot of the blank solution, the LOD can be determined to be $1.0 \mathrm{mg} / \mathrm{l}$ at the laser energy of $23 \pm 1 \mathrm{~mJ}$. The resulting LOD of $\mathrm{Na}$ sample can be improved with increasing the laser energy [22].

\subsection{Interpretation of exemption from matrix interference}

In this work, the added matrices, each with the same $\mathrm{K}^{+}$cation but different anions, seem not to cause any marked interference with the LIB/C correlated signals of the analyte solution alone. Such findings of resistance of the matrix interference may be reasonably interpreted in the following. When the pulse energy is large enough to ionize the droplets, the current collected is substantially from the electrons, which move more rapidly than the ions and are in a much shorter distance toward one

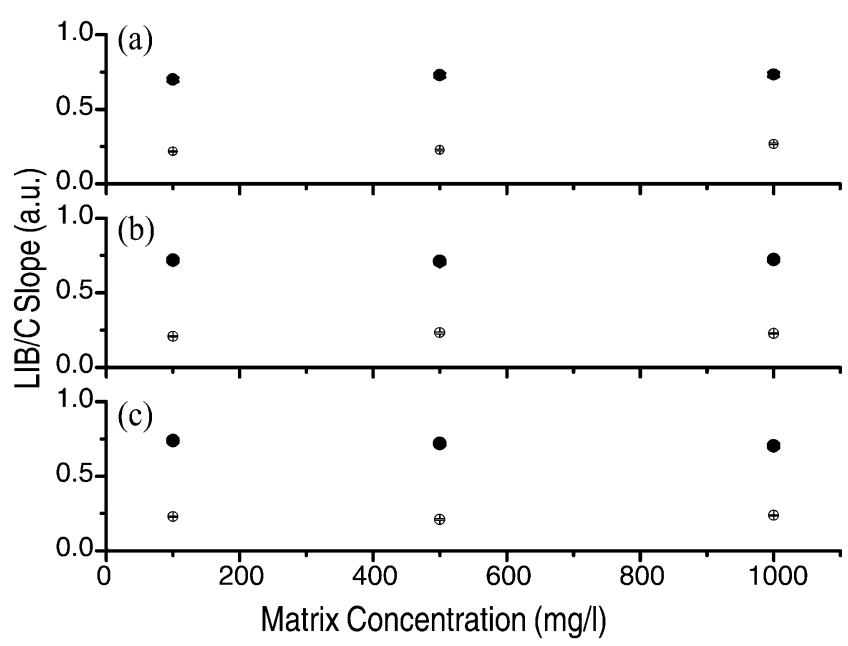

Fig. 4. The slopes in the LIB/C correlation plots of $200 \mathrm{mg} / 1 \mathrm{Na}$ concentration $(\bigcirc)$ and blank solution $(\bigcirc)$, respectively, as a function of the 100,500 , and $1000 \mathrm{mg} / 1$ matrix salt concentration. Matrix salts added to the $\mathrm{NaCl}$ or blank solutions are (a) $\mathrm{KH}_{2} \mathrm{PO}_{4}$, (b) $\mathrm{KCl}$, and (c) $\mathrm{KNO}_{2}$. A small error bar indicates the standard deviation $(1 \sigma)$ of slope in the correlation plot.

end of the collector $[23,24]$. The electrons result mainly from the ionized water/methanol droplets and their fragments, while little from the contained sample salt. Such polyatomic molecules used as the solvent may lead to the electron multiplication as fragmented and ionized [22]. Like the sample salt, the electron contributions from the added matrix may be negligible, as compared to those from the water/methanol droplets. This consequence is demonstrated in Fig. 6, showing that 200 shots of current signals, each acquired from either an analyte plus matrix solution or a water/ methanol solvent, are distributed randomly in the same intensity range. The fact is also reflected in the coordinate of current signal in Fig. 3, in which the current

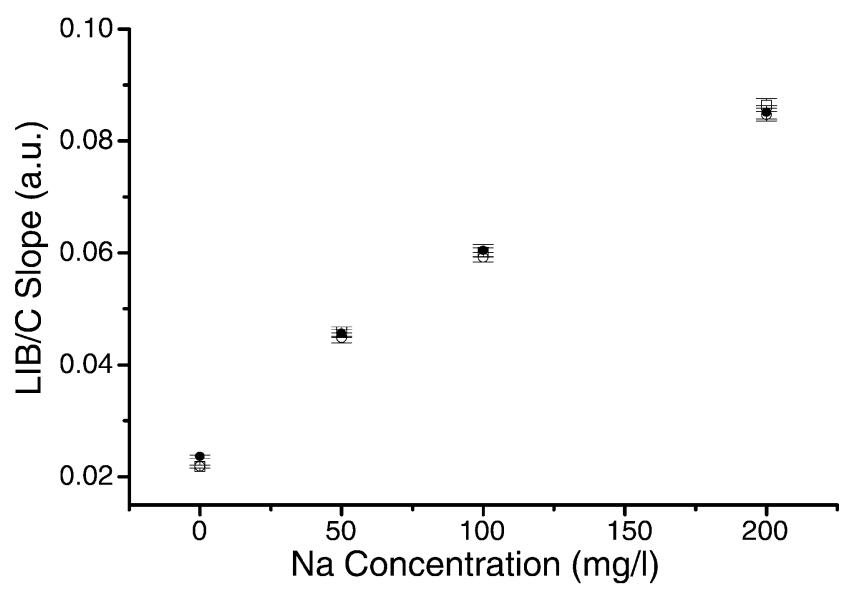

Fig. 5. Calibration curves of $\mathrm{NaCl}$ solution with a concentration of 0 $(\bigcirc), 1000(\bullet)$, and $2000(\square) \mathrm{mg} / \mathrm{l} \mathrm{KCl}$ added as matrix. 


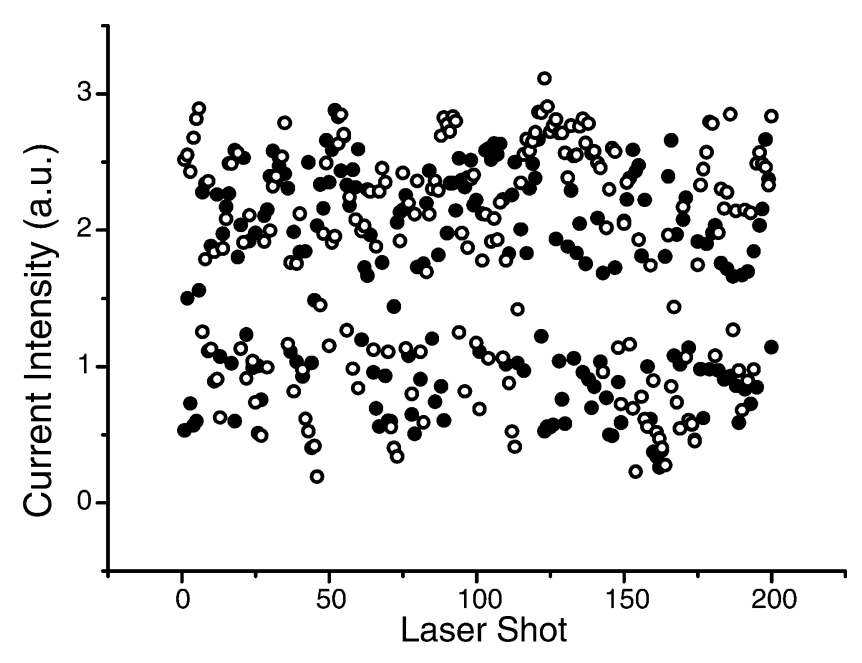

Fig. 6. Distributions of 200-shots current signals. $(\bigcirc)$ denotes a solution of $200 \mathrm{mg} / 1 \mathrm{Na}$ concentration with $1000 \mathrm{mg} / 1 \mathrm{KNO}_{2}$ added as matrix, and (-) denotes a water/methanol solvent alone.

range remains almost the same, independent of variation of the sample concentrations.

Since the atomized sample may be surrounded in an electron-rich atmosphere from ionization of the polyatomic solvent, the relatively less electron addition from the matrix may not result in significant equilibrium shift of the ionization process. Thus, the ionization interference caused by the matrix may not enhance the LIB emission. In addition, the atomized sample is also weakly affected by the chemical interference, since the formed compounds may be feasibly decomposed in the generation process of very high temperature plasma. Accordingly, the calibration curve and the subsequent LOD determination are not deviated by a large amount of matrix salt added.

In addition to tolerance of matrix interference, the LIB/C correlated analysis greatly helps reduce the signal fluctuation. The obtained current response is expected to be proportional to both the ablated droplet sizes and the electron motion, but irrespective of the salt concentration contained. The larger the droplet size is ablated, the more the electron amounts (or the current response) might be probably induced. Meanwhile, a higher plasma excitation temperature might speed up the electron motion to cause a larger current response. In a following work, we attempt to verify that the current normalization method should have probably taken into account both the ablated amount of the sample and the plasma excitation temperature [25]. In contrast, continuum background emission is usually adopted as an internal reference to improve the signal quality. Nevertheless, this method might lack to consider the plasma excitation temperature $[11,25]$.

\section{Conclusion}

We have inspected the matrix influence on the LIBS analysis of the $\mathrm{Na}$ sample. The microdroplets of the mixed solution were generated via an electrospray ionization needle. In spite of a smaller amount of sample ablated, the resultant LIB emission signal is well correlated linearly with the corresponding plasma-induced current. In this manner, the obtained calibration curve yields the $\mathrm{Na}$ LOD value approximately $1 \mathrm{mg} / \mathrm{l}$, as comparable as those reported previously. In the LIBS analysis, the electron contributions generated by the matrix salts are negligible in an electron-rich atmosphere. The matrix effect studied in this work shows insignificant influence on the accuracy of the elemental analysis by LIBS with the current normalization. The LIB/C normalized signal might have probably taken into account both the ablated weight of the sample and the plasma excitation temperature. This method is capable of reducing signal fluctuation, resisting matrix interference, and achieving a lower LOD.

\section{Acknowledgments}

This work is supported by the National Science Council of the Republic of China under Contract No. NSC91-2113-M-002-033.

\section{References}

[1] L.J. Radziemski, T.R. Loree, D.A. Cremers, N.M. Hoffman, Time-resolved laser-induced breakdown spectrometry of aerosols, Anal. Chem. 55 (1983) 1246-1252.

[2] Y.-I. Lee, K. Song, J. Sneddon, in: J. Sneddon, T.L. Thiem, Y.-I. Lee (Eds.), Lasers in Analytical Atomic Spectroscopy, VCH, New York, 1997, pp. 197-235.

[3] U. Panne, R.E. Neuhauser, C. Haisch, H. Fink, R. Niessner, Remote analysis of a mineral melt by laser-induced plasma spectroscopy, Appl. Spectrosc. 56 (2002) 375-380.

[4] J.A. Bolger, Semi-quantative laser-induced breakdown spectroscopy for analysis of mineral drill core, Appl. Spectrosc. 54 (2000) 181-189.

[5] D. Kossakovski, J.L. Beauchamp, Topographical and chemical microanalysis of surfaces with a scanning probe microscope and laser-induced breakdown spectroscopy, Anal. Chem. 72 (2000) 4731-4737.

[6] C.M. Davies, H.H. Telle, D.J. Montgomery, R.E. Corbett, Quantitative-analysis using remote laser-induced breakdown spectroscopy (LIBS), Spectrochim. Acta Part B 50 (1995) 1059-1075.

[7] N.H. Cheung, E.S. Yeung, Single-shot element analysis of liquids based on laser vaporization at fluences below breakdown, Appl. Spectrosc. 47 (1993) 882-886.

[8] L. Xu, V. Bulatov, V.V. Gridin, I. Schechter, Absolute analysisof particulate materials by laser-induced breakdown spectroscopy, Anal. Chem. 69 (1997) 2103-2108.

[9] C. Aragon, J.A. Aguilera, F. Penalba, Improvements in quantitative analysis of steel composition by laser-induced breakdown spectroscopy at atmospheric pressure using an infrared Nd: YAG laser, Appl. Spectrosc. 53 (1999) 1259-1267. 
[10] D.A. Cremers, J.E. Barefield II, A.C. Koskelo, Remote elemental analysis by laser-induced breakdown spectroscopy using a fiber-optic cable, Appl. Spectroc. 49 (1995) 857-860.

[11] I.B. Gornushkin, B.W. Smith, G.E. Potts, N. Omenetto, J.D. Winefordner, Considerations on the correlation between signal and background in laser-induced breakdown spectroscopy using single-shot analysis, Anal. Chem. 71 (1999) 5447-5449.

[12] U. Panne, C. Haisch, M. Clara, R. Niessner, Analysis of glass and glass melts during the vitrification process of fly and bottom ashes by laser-induced plasma spectroscopy. Part I: normalization and plasma diagnostics, Spectrochim. Acta Part B 53 (1998) 1957-1968.

[13] E.H. van Veen, M.T.C. de Loos-Vollebregt, On the use of line intensity ratios and power adjustments to control matrix effects in inductively coupled plasma optical emission spectrometry, J. Anal. At. Spectrom. 14 (1999) 831-838.

[14] A.S. Eppler, D.A. Cremers, D.D. Hickmott, M.J. Ferris, A.C. Koskelo, Matrix effects in the detection of $\mathrm{Pb}$ and $\mathrm{Ba}$ in soils using laser-induced breakdown spectroscopy, Appl. Spectrosc. 50 (1996) 1175-1181.

[15] C. Chaleard, P. Mauchien, N. Andre, J. Uebbing, J.L. Lacour, C. Geertsen, Correction of matrix effects in quantitative elemental analysis with laser ablation optical emission spectrometry, J. Anal. At. Spectrom. 12 (1997) 183-188.

[16] H.J. Hakkanen, J.E.I. Korppi-Tommola, Laser-induced plasma emission spectrometric study of pigments and binders in paper coatings: matrix effects, Anal. Chem. 70 (1998) 4724-4729.
[17] S.I. Gornushkin, I.B. Gornushkin, J.M. Anzano, B.W. Smith, J.D. Winefordner, Effective normalization technique for correction of matrix effects in laser-induced breakdown spectroscopy detection of magnesium in powdered samples, Appl. Spectrosc. 56 (2002) 433-436.

[18] S.J. Gaskell, Electrospray: principles and practice, J. Mass Spectrom. 32 (1997) 677-688.

[19] J.F. Banks, Recent advances in capillary electrophoresis electrospray mass spectrometry, Electrophoresis 18 (1997) $2255-2266$.

[20] M.G. Ikonomou, A.T. Blades, P. Kebarle, Electrospray-ion spray: a comparision of mechanisms and performance, Anal. Chem. 63 (1991) 1989-1998.

[21] Z. Olumee, J.H. Callahan, A. Vertes, Droplet dynamics changes in electrostatic sprays of methanol-water mixtures, J. Phys. Chem. A 102 (1998) 9154-9160.

[22] J.S. Huang, C.B. Ke, L.S. Huang, K.C. Lin, The correlation between ion production and emission intensity in the laserinduced breakdown spectroscopy of liquid droplets, Spectrochim. Acta Part B 57 (2002) 35-48, and references therein.

[23] G.C. Turk, Imaging the active flame volume for pulsed laserenhanced ionization spectroscopy, Anal. Chem. 64 (1992) 1836-1839.

[24] C.B. Ke, K.C. Lin, Laser-enhanced ionization detection of $\mathrm{Pb}$ in seawater by flow injection analysis with on-line preconcentration and separation, Anal. Chem. 71 (1999) 1561-1567.

[25] J.S. Huang, K.C. Lin, to be published. 